Available online at GSC Online Press Directory

GSC Biological and Pharmaceutical Sciences

e-ISSN: 2581-3250, CODEN (USA): GBPSC2

Journal homepage: https://www.gsconlinepress.com/journals/gscbps

(CASE REPORT)

\title{
61-year-old man with intestinal amyloidosis: A case report
}

\author{
Erra Stefania *, Zambello Luca, Frigeri Alessia, De Luca Michele and Caminiti Valentina \\ Surgical Pathology Department, Santo Spirito Hospital, Casale Monferrato, Viale Giolitti 2, Italy.
}

Publication history: Received on 05 June 2020; revised on 16 June 2020; accepted on 19 June 2020

Article DOI: https://doi.org/10.30574/gscbps.2020.11.3.0164

\begin{abstract}
Gastrointestinal amyloidosis (GIA), a protein deposition disorder, represents a complex common pathway that encompasses multiple etiologies and presentations. It is characterized by accumulation of insoluble extracellular protein fragments that have been rendered resistant to digestion. The most common form of systemic amyloidosis in the Western world is $\mathrm{AL}$, in which the fibrils derive from monoclonal immunoglobulin light chains. Its incidence is approximately 10 cases / million / year with an average age at diagnosis of around 60 years. Herein, we report a case of 61-year-old man with colic AL amyloidosis.
\end{abstract}

Keywords: Amyloidosis; Protein deposition; Colon; Monoclonal immunoglobulin

\section{Introduction}

Amyloidosis is a group of diseases caused by extracellular deposition of amyloid fibrils leading to organ dysfunction. Amyloid consists of misfolded, insoluble, toxic peptide aggregates, which are oriented in a $\beta$-sheet structures.[1] [2]

GIA can be acquired or genetic, and most commonly results from chronic inflammatory disorders (AA amyloidosis), hematologic malignancy (AL amyloidosis), and end-stage renal disease ( $\beta-2$ amyloidosis).The acquired form of amyloidosis results most commonly from a hyperproteinemic state originating from either plasmas cell dyscrasia (AL) or a chronic inflammatory state. [3]

Within the GI tract, amyloid deposition occurs in the muscularis mucosae, within close proximity to vasculature, nerves, and nerve plexuses. [4] Common features of GIA are represented by the frailty of blood vessels, hinders intrinsic peristalsis and decreases the compliance of the gut wall. [5]

Differently from how it was initially considered, the substance is not amorphous, but has a $\beta$-sheet structure, which is why the term $\beta$-fibrillosis is preferred for the disease. It is deposited in the extracellular site in various organs, causing damage to the parenchyma.

Amyloid is composed of $2 \beta$-sheet helices associated with complex carbohydrates and the SAP protein. The fibrils have a very different chemical composition since the amyloidogenic peptide which represents the outermost part of them (equal to 95\%) derives from the deposition of a different precursor protein according to the pathology in question, which assumes an insoluble $\beta$-fibrillar conformation. This can happen either by a mutation to the protein gene, which thus assumes an incorrect conformation, or by the action of a protease that leads to incorrect cleavage.

Amyloidosis AL is characterized by an abnormal production of immunoglobulins, which are phagocytosed by macrophages and digested by their proteases. It is therefore possible to form proteolytic fragments of the $\kappa$ and $\lambda$ light

\footnotetext{
* Corresponding author: Erra Stefania
}

Copyright (C) 2020 Author(s) retain the copyright of this article. This article is published under the terms of the Creative Commons Attribution Liscense 4.0. 
chains, corresponding to the hypervariable region, able to form $\beta$-sheet fibrils and to bind heparan sulfate and AP substance.

The deposition of amyloid occurs mainly at the level of: tongue (macroglossia, with difficulty speaking and swallowing), nerves (peripheral neuropathies), heart (restrictive cardiomyopathy), joints (arthropathies), skin (skin nodules) and intestine (malabsorption).

It is associated with multiple myeloma, where there is amyloid in 6-15\% of cases, to Waldenström's macroglobulinemia and to malignant lymphoma B. 50\% of deaths for this type of amyloid are associated with restrictive myocardiopathy. There are also foamy urines, due to the presence of Ig, or Bence-Jones Proteinuria, associated with multiple myeloma. [6]

Common clinical aspects include the presence of a proteinuria often in the nephrotic environment with or without renal insufficiency, a restrictive cardiomyopathy with diastolic dysfunction that leads to severe congestive heart failure, an idiopathic sensory peripheral neuropathy (15\% of cases), a carpal tunnel syndrome, a gastrointestinal involvement responsible for diarrhea with malabsorption or gastrointestinal haemorrhage and hepatosplenomegaly. More than half of AL amyloidosis patients at the presentation have a clinical involvement of two or more organs. [1]

\section{Case report}

A 61-year old man underwent to hospital for gastointestinal symptoms, as weight loss, diarrhea, abdominal pain, malabsorption and esophageal reflux. He didn't report familiar with colon cancer and no fecal occult blood found by the FOBT examination. He was subjected to a total colonoscopy showing numerous diverticula in the visceral lumen; on the colonic left flexure a micropolyp was detected and removed with the forceps; other two peduncolated polyps in the ascendant colon and in the distal sigma were removed by electrocautery.

The formalin fixed specimens were sentto the surgical pathology service in Casale Monferrato hospital.Histologic examination of the splenic flexure biopsy showed fragments of colic mucosa with focal low-grade adenomatous dysplasia of the glandular elements; the sigma biopsy corresponds to tubulovillous adenoma with low-grade dysplasia, the right colon biopsy shows fragments of colic mucosa with normostructured glandular elements and the presence of birefringent amorphous substance in polarized light, referable to amyloid and intermingled with glands.

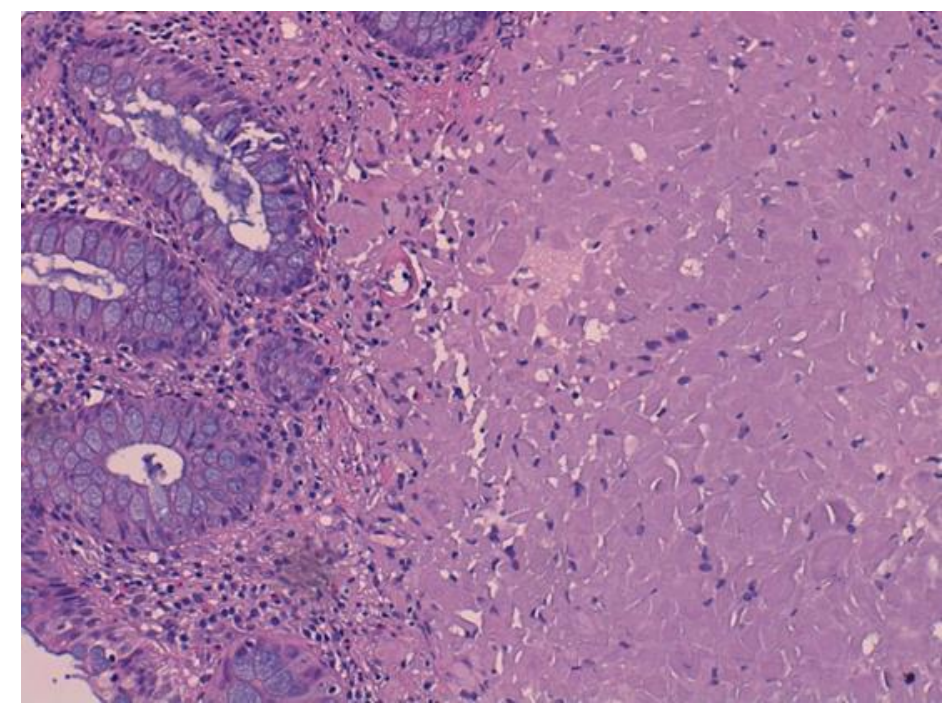

Figure 1 Hematoxylin and eosin staining of intestinal histological examination: homogeneous eosinophilic material, attributable to amyloid in the vessel wall of submucosa (Magnification 10X) 


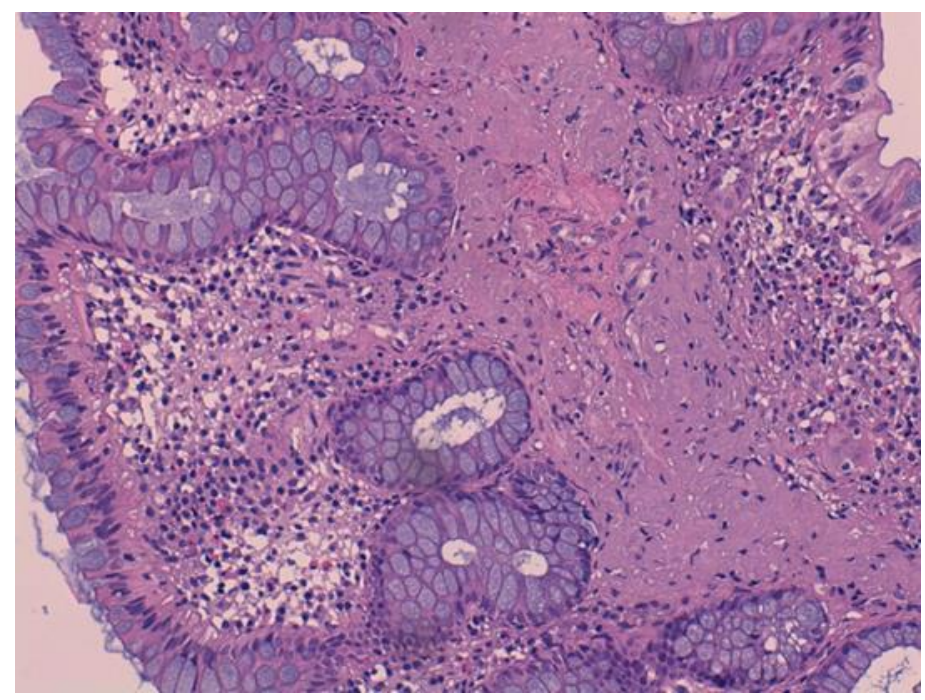

Figure 2 Mucosal and submucosal layers with a deposit of birefringent amorphous substance in polarized light, referable to amyloid. (Magnification 10X)

\section{Discussion}

Amyloidosis is a systemic disorder caused by deposition of insoluble abnormal amyloid fibrils interfering with tissues functionality. The type of the protein is misfolded and the organ or tissue in which they are deposited determine the clinical manifestation of amyloidosis. [7]

The global incidence of systemic amyloidosis is about 8-12 per million person per year. The initial manifestation can be non-specific, such as weight loss or fatigue. Gastrointestinal symptoms are relatively uncommon, occurring in less than $1 \%$ of patients even though post-mortem gastrointestinal involvement can be seen in up to $40 \%$ of cases. [8]

The gold standard for diagnosing amyloidosis is represented from histological examination of tissue biopsy of an affected organ in whose context Congo red stain can detect green birefringence under polarized light. [9] Those presenting with gastrointestinal manifestations often have indications for endoscopy such as weight loss, dyspepsia refractory to medical therapy or gastrointestinal bleeding. [10] Any site can be affected, but the small bowel is most commonly affected. [11]

The biopsy of colon amyloidosis stained with hematoxylin an eosin shows inflammatory cells, infiltration, primarily of the plasma cells and amyloid deposition.

Amyloidosis with evidence of organ involvement is an indication for treatment of any monoclonal gammopathy regardless of clone burden. These patients benefit from oncology consultation for consideration of chemotherapy and autologous stem cells transplant. [12]

Amyloidosis typically is associated with a worse prognosis due to its association with underlying and occult malignancy.

\section{Conclusion}

Amyloidosis involving the gastrointestinal tract encompasses a wide variety of symptoms, often non-specific, which range from nausea, reflux, diarrhea, to more severe manifestations such as hemorrhage and obstruction. Symptoms of enteric amyloidosis should not be undervalued because they can represent the initial manifestation of hematologic disease, chronic infection and autoimmune disease, as well as paraneoplastic expression. Attention must be given to family history and knowledge of conditions that predispose to amyloid formation. 


\section{Compliance with ethical standards}

\section{Disclosure of conflict of interest}

Authors declare that there are no conflicts of interest in connection with this paper, and the material described is not under publication or consideration for publication elsewhere.

\section{Statement of informed consent}

Informed consent has been obtained from the subject included in the study.

\section{References}

[1] Merlini G and Bellotti V. (2003). Molecular mechanisms of amyloidosis. N. Engl. J. Med., 349, 583- 596.

[2] Rowe K, Pankow J, Nehme F and Salyers W. (2017). Gastrointestinal Amyloidosis: Review of the Literature. Cureus, 9(5), e1228.

[3] Kaiserling E and Krober S. (1995). Massive intestinal hemorrhage associated with intestinal amyloidosis. An investigation of underlying pathologic processes. Gen Diagn Pathol, 141, 147-154.

[4] Hirschfield GM. (2004). Amyloidosis: a clinico-pathophysiological synopsis. Semin. Cell Dev. Biol, 15, 39-44.

[5] Gertz MA, Lacy MQ and Dispenzieri A.(1999). Amyloidosis. Hematol. Oncol. Clin. North Am, 13, 1211- 1233.

[6] Lee ASY, Lee DZQ and Vasanwala FF. (2016). Amyloid light-chain amyloidosis presenting as abdominal bloating: a case report. J. Med. Case Rep, 10, 68.

[7] Rocken C, Saeger W and Linke RP. (1994). Gastrointestinal amyloid deposits in old age. Report on 110 consecutive autopsical patients and 98 retrospective bioptic specimens. Pathol. Res. Pract, 190, 641-649.

[8] Katoh N, Matsuda M and Ikeda S. (2015). Clinical, endoscopic, and histopathological features of localized immunoglobulin light chain (AL) amyloidosis in the gastrointestinal tract. Amyloid, 22, 254-256.

[9] Lam PW, Lam YS, Liu HW, et al. (2016). Gastrointestinal: A rare cause of bloody diarrhoea - Gastrointestinal amyloidosis. J Gastroenterol Hepatol, 31, 1913.

[10] Syed U, Ching Companioni RA, Alkhawam H, et al. (2016). Amyloidosis of the gastrointestinal tract and the liver: clinical context, diagnosis and management. Eur. J. Gastroenterol. Hepatol, 28, 109-121.

[11] Dispenzieri A, Buadi F, Kumar SK, et al. (2015).Treatment of immunoglobulin light chain amyloidosis: mayo stratification of myeloma and risk-adapted therapy (msmart) consensus statement. Mayo Clin Proc, 90, 10541081.

\section{How to cite this article}

Erra S, Zambello L, Frigeri A, De Luca M and Caminiti V. (2020). 61-year-old man with intestinal amyloidosis: A case report. GSC Biological and Pharmaceutical Sciences, 11(3), 185-188. 